\title{
The ZEPLIN-III dark matter detector: Performance study using an end-to-end simulation tool
}

H.M. Araújo a,b,*, D.Yu. Akimov ${ }^{\text {c }}$, G.J. Alner ${ }^{\text {b }}$, A. Bewick ${ }^{\text {a }}$, C. Bungau ${ }^{\text {b }}$, B. Camanzi ${ }^{b}$, M.J. Carson e , V. Chepel d, H. Chagani e, D. Davidge a, J.C. Davies e , E. Daw e, J. Dawson a , T. Durkin b, B. Edwards ${ }^{\text {a,b }}$, T. Gamble e , C. Ghag ${ }^{\text {f }}$, R. Hollingworth ${ }^{\mathrm{e}}$, A.S. Howard ${ }^{\mathrm{a}}$, W.G. Jones ${ }^{\text {a }}$, M. Joshi ${ }^{\text {a }}$, J. Kirkpatrick ${ }^{\mathrm{e}}$, A. Kovalenko ${ }^{\text {c }}$, V.A. Kudryavtsev ${ }^{\mathrm{e}}$, V.N. Lebedenko ${ }^{\text {a }}$, T. Lawson ${ }^{\mathrm{e}}$, J.D. Lewin ${ }^{\mathrm{b}}$, P. Lightfoot ${ }^{\mathrm{e}}$, A. Lindote ${ }^{\mathrm{d}}$, I. Liubarsky ${ }^{\mathrm{a}}$, M.I. Lopes ${ }^{\mathrm{d}}$, R. Lüscher ${ }^{\mathrm{b}}$, P. Majewski ${ }^{\mathrm{e}}$, K. Mavrokoridis ${ }^{\mathrm{e}}$, J. McMillan ${ }^{\mathrm{e}}$, B. Morgan ${ }^{\mathrm{e}}$, D. Muna ${ }^{e}$, A.S. Murphy ${ }^{f}$, F. Neves ${ }^{d}$, G. Nicklin ${ }^{e}$, S. Paling e , J. Pinto da Cunha ${ }^{d}$, S. Plank ${ }^{\text {f }}$, R. Preece ${ }^{b}$, J.J. Quenby ${ }^{\text {a }}$, M. Robinson ${ }^{\text {e }}$, C. Silva ${ }^{d}$, V.N. Solovov ${ }^{d}$, N.J.T. Smith ${ }^{\text {b }, \text { P.F. Smith }}{ }^{\text {b }}$, N.J.C. Spooner ${ }^{\text {e }}$, V. Stekhanov ${ }^{\text {c }}$, T.J. Sumner ${ }^{\text {a }}$,

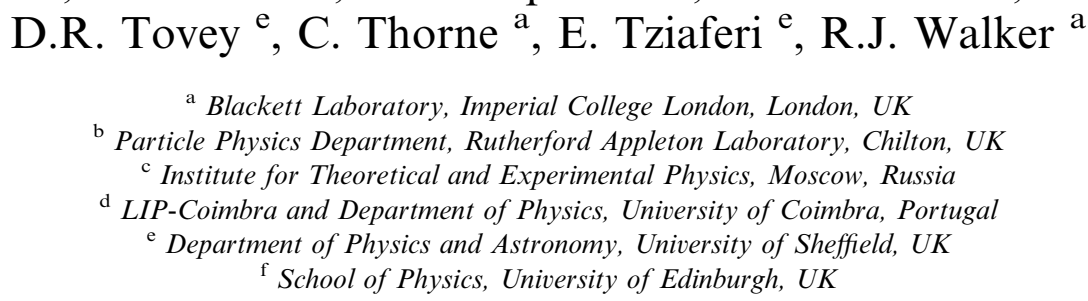

Received 13 March 2006; received in revised form 22 May 2006; accepted 28 May 2006

Available online 30 June 2006

\begin{abstract}
We present results from a GEANT4-based Monte Carlo tool for end-to-end simulations of the ZEPLIN-III dark matter experiment. ZEPLIN-III is a two-phase detector which measures both the scintillation light and the ionisation charge generated in liquid xenon by interacting particles and radiation. The software models the instrument response to radioactive backgrounds and calibration sources, including the generation, ray-tracing and detection of the primary and secondary scintillations in liquid and gaseous xenon, and subsequent processing by data acquisition electronics. A flexible user interface allows easy modification of detector parameters at run time. Realistic datasets can be produced to help with data analysis, an example of which is the position reconstruction algorithm developed from simulated data. We present a range of simulation results confirming the original design sensitivity of a few times $10^{-8} \mathrm{pb}$ to the WIMP-nucleon cross-section.
\end{abstract}

(C) 2006 Elsevier B.V. All rights reserved.

PACS: 21.60.Ka; 29.40.Mc; 95.35.+d; 14.80.Ly

Keywords: ZEPLIN-III; GEANT4; Liquid xenon; Radiation detectors; Dark matter; WIMPs

\footnotetext{
* Corresponding author. Address: Astrophysics Group, Department of Physics, Blackett Laboratory, Imperial College London, Prince Consort Road, London SW7 2BW, UK. Tel.: +44 20759 47549; fax: +44 2075947541.

E-mail address: H.Araujo@imperial.ac.uk (H.M. Araújo).
} 


\section{Introduction}

ZEPLIN-III is a two-phase (liquid/gas) xenon detector developed by the UK Dark Matter Collaboration and international partners, ${ }^{1}$ which will try to identify and measure galactic dark matter in the form of Weakly Interacting Massive Particles, or WIMPs [1,2]. Upon completion of physics testing now underway at Imperial College London, the system may join the ZEPLIN-II [3] and DRIFT-IIa [4] experiments already operating $1100 \mathrm{~m}$ underground in our laboratory at the Boulby mine (North Yorkshire, UK).

Two-phase emission detectors based on the noble gases date back several decades [5], but this technology has gained momentum since the UKDM Collaboration first explored the potential of high-field xenon systems [6-8]. The operating principle is that different particle species generate relatively different amounts of scintillation light and ionisation charge in liquid xenon (LXe). The ratio between these two signal channels provides a powerful technique to discriminate between electron and nuclear recoil interactions. WIMPs are expected to scatter elastically off $\mathrm{Xe}$ atoms, much like neutrons, and the recoiling nucleus will produce a different signature to that of $\gamma$-ray interactions and other sources of electron recoils.

WIMP detectors differ from more traditional detectors of nuclear radiation in that they require (i) extremely low radioactive and cosmic-ray backgrounds, addressed by the use of radio-pure materials and operation deep underground; (ii) excellent discrimination of the remaining background events, hopefully better than 1000:1 rejection efficiency for electron recoils; (iii) a low energy threshold for nuclear recoils, since the kinematics of WIMP-nucleus scattering results in a very soft recoil spectrum $(\lesssim 100 \mathrm{keV})$.

Monte Carlo simulations are an essential tool in devising an experimental strategy capable of tackling these issues. Acceptable levels of trace contamination must be assessed for all detector materials, requiring simulations of local backgrounds expected from each component. Cosmic-rayinduced backgrounds also need careful calculation, since experimental measurements would require nothing short of a dedicated WIMP detector. Having established the residual electron/photon and neutron event rates, the level of discrimination and energy threshold which can realistically be achieved must be calculated with the help of detailed detector simulations - and possibly fed back to the design process. In addition, the data produced by twophase detectors are often complex, and particular simulations are required to help extract actual physics parameters. Finally, realistic datasets help with planning the data acquisition electronics and the data analysis software.

In this paper we describe a simulation tool used throughout this process [9], based on the GEANT4 Monte Carlo toolkit [10]. The package builds upon previous sim-

\footnotetext{
${ }^{1}$ Edinburgh University, Imperial College London, ITEP-Moscow, LIPCoimbra, Rutherford Appleton Laboratory and Sheffield University.
}

ulation work [11-13] and experimental measurements with high-field, two-phase prototype detectors [7,8]. After overviewing the detector and the software, a description is given of the simulation models used to calculate the detector response. Simulation results are then presented for the dominant background contributions, calibration runs with $\gamma$ and neutron sources, and position reconstruction capabilities, leading to a predicted performance of the instrument as a WIMP detector.

\subsection{The ZEPLIN-III detector}

The xenon vessel containing the WIMP target is housed in a 1-m tall vacuum cryostat, on top of a liquid nitrogen reservoir which cools it to around $-100{ }^{\circ} \mathrm{C}$. We refer to Fig. 1 for the model representation of the xenon chamber. All major metal components of the detector are made from high-purity C103 copper. Inside the xenon vessel an array of 31 2-in. photo-multiplier tubes (PMTs) is immersed in the liquid phase, looking up to a $\simeq 40$-mm thick LXe disk topped by a 5-mm layer of gas. Up to $40 \mathrm{kV}$ can be applied between a polished copper plate covering the gas phase (hereafter 'anode mirror') and a metal wire grid located $35 \mathrm{~mm}$ below the liquid surface ('cathode grid'), defining the active region of the detector. A second wire grid ('PMT grid') placed just above the array $(5 \mathrm{~mm}$ below the cathode) defines a reverse field region which suppresses secondary signals from low-energy photons from the PMTs and helps protect the PMT photo-cathodes from stray electric fields. The PMTs, arranged in a closely-packed hexagonal array, are encased in 2-in. holes bored in a copper screen in order to prevent optical cross-talk. A lower plate ('PMT mirror') covers the array; this mirror has conical cuts around the PMT windows intended to improve the light collection and prevent the escape and detection of stray light generated around the PMT bodies. The diameter of the active LXe volume is approximately $40 \mathrm{~cm}$, while that of the PMT array is $34 \mathrm{~cm}$. The array is powered by a network of thin copper plates located inside the xenon vessel, which provides common connection to each dynode on all the PMTs and hence reduces the number of required feedthroughs quite considerably. A detailed description of the detector construction is given elsewhere [14].

When a particle interacts in LXe, VUV scintillation light is promptly produced. In addition, a strong electric field (up to $\sim 8 \mathrm{kV} / \mathrm{cm}$ ) prevents most of the ionisation charge produced around the particle track from recombining. The extracted charge carriers drift upward to the liquid surface, and are emitted into the gas phase. Here, the strong electric field (double that of the liquid phase) leads to the production of further VUV photons, by the process known as electroluminescence or proportional scintillation. Therefore, both scintillation and ionisation signals give rise to optical signatures which are detected with the same PMT array, the time separation between them being proportional to the vertical $(z)$ coordinate. We label these signals as the "primary' and the 'secondary', or S1 and S2, respectively. The 

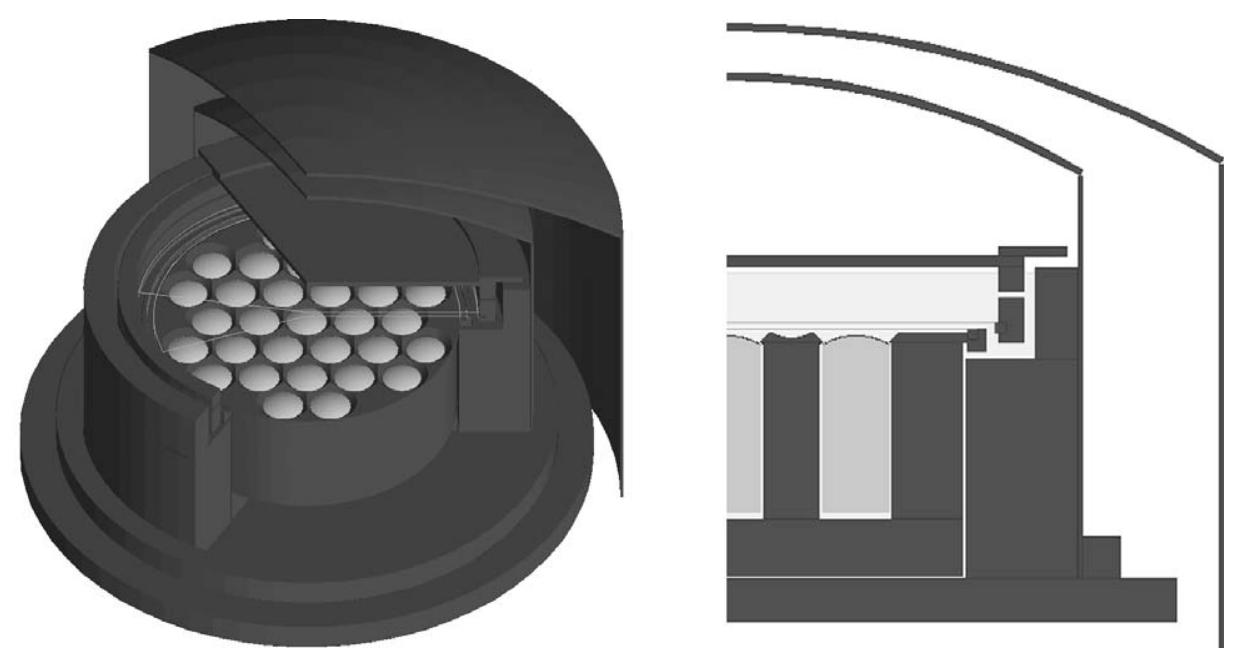

Fig. 1. Cut-out (left) and cross-sectional (right) views of the GEANT4 implementation of the 'full' ZEPLIN-III geometry.

ratio $\mathrm{S} 2 / \mathrm{S} 1$ is higher for electrons than nuclear recoils. In low-energy atom-atom collisions, most of the energy loss goes into recoil of the nuclei and only a small fraction converts into electronic loss (i.e., electron excitation/ionisation). Electron $/ \gamma$-ray interactions act directly on the outer atomic electrons, yielding more ionisation charge.

The 31 PMT signals are fed into wideband amplifiers and split into a dual-range data acquisition system (DAQ). A large dynamic range ensures sensitivity to very small primaries containing only a few photo-electrons (phe) as well as large secondaries without saturation. All 62 channels are sampled at $500 \mathrm{MS} / \mathrm{s}$ by 8-bit digitisers.

\subsection{The modelling software design}

The main requirements underlying the software design were (i) that it should model the transport and interactions of particles internal and external to the detector, down to the production of electron and nuclear recoils; (ii) simulate the physical processes involved in the generation of the optical response to scintillation and electroluminescence; (iii) generate the electrical response (digitised voltage timelines) for all channels in order to produce realistic datasets; (iv) operating parameters should be easily modified by the user, in particular the LXe height and the applied electric field; (v) it should be user friendly.

GEANT4 is arguably the most comprehensive toolkit of its kind, and the only one to fulfill the above requirements. Besides an extensive list of physics models, its modular and transparent design means that new ones can be easily added (e.g., electroluminescence). Its flexibility allows further processing of simulated events (DAQ, event display, data analysis, etc.). Versatile specification of the primary particle generator means that individual particles, radioisotopes and sources with complex spatial and energy distributions can be specified at run time. Finally, userinterfaces are easily created, allowing the user to control most physics parameters without having to delve into the code itself. This is important since many optical and charge transport properties are still ill-defined, and different parameter combinations have to be assessed. In this package, primary generator, detector and physics parameters can be set interactively by commands and macros available for many simulation tasks.

\section{The simulation model}

\subsection{GEANT4 solid model}

Two geometry representations have been set up. The one shown in Fig. 1 includes most detector components located above the liquid nitrogen vessel. A second geometry implements only the (optically) active region, comprised between the PMT photo-cathodes and the anode mirror. Less computationally demanding than the full model, this is useful for light collection and similar studies. Both produce exactly the same optical response. The LXe height, chosen by default to be $35 \mathrm{~mm}$ above the cathode (leaving $5 \mathrm{~mm}$ of gas below the anode mirror) can also be set between runs. This entails modifying the electric field distribution as well as optical and other properties, as explained below. Additional external components will be included at a future stage, namely a scintillator veto system surrounding the detector as well as hydrocarbon and lead shielding for external neutrons and $\gamma$-rays, respectively.

\subsection{The optical model}

Optical ray-tracing in the detector takes into account the optical constants of liquid and gaseous xenon, the angular reflectivities of copper surfaces and electrode grids, and the optical and detection properties of the PMTs, all of which are defined at the xenon scintillation wavelength of $\sim 175 \mathrm{~nm}(7 \mathrm{eV})$.

For LXe, a refractive index $n=1.69$ and an attenuation length of $36.4 \mathrm{~cm}$ were considered [15]. Although the latter 
is probably dominated by Rayleigh scattering (c.f. Ref. [16], as well as preliminary results from our ZEPLIN-II detector), only photo-absorption was considered here the difference in light collection is relatively small.

The angular reflectivity of copper is quite uncertain at VUV wavelengths, depending on the surface finish, oxidation state and possible LXe condensation onto the cold surfaces. A single measurement was found in the literature, indicating $R=27 \%$ for normal incidence for a clean-cut surface [17]; a more conservative $15 \%$ was adopted instead. The GEANT4 'unified' model [18] was chosen to treat the angular reflection from metal surfaces; the parameter $\sigma_{\alpha}$, which characterises the Gaussian smearing of the exit angle in this model, was set to $20^{\circ}$, producing a half-width $\approx 45^{\circ}$ for normal incidence. We point out that the choice of model is not informed by experimental data - lacking for many materials for this wavelength range. A high-reflectivity case ( $R=90 \%$ for anode and PMT mirrors) was also considered, to assess the benefit of electroplating the mirrors in a future upgrade.

The two electrode grids (wire-wound) are implemented as continuous dielectric sheets with refractive index matched to that of LXe (no Fresnel reflection) but with an absorption length chosen to give $10 \%$ absorption at normal incidence (equal to the grid wire/pitch ratio). The dielectric absorption mimics the obscuration of light with angle of incidence to good approximation for all but the largest angles with respect to the grid normal.

The PMT models include a curved quartz window supporting an opaque photo-cathode with a user-defined quantum efficiency $(\mathrm{QE})$. A photon is detected as a photoelectron depending on the outcome of a random throw. Since the photon attenuation coefficient increases with decreasing wavelength for typical photo-cathode materials, the assumption of an optically-thick photo-cathode should hold in the VUV. Even though no VUV-specific information could be found for the reflectivity of the photo-cathode layer itself, we believe that a black model is reasonable at short wavelengths - although this is not so in the visible [19]. Also, it should be noted that manufacture-quoted QEs are usually measured in air or vacuum. A slightly better optical match is obtained when PMTs are immersed in LXe due to the similar refractive indices $(n=1.6$ for quartz). However, given that other losses are also considered (e.g., window absorption and reflection from metal fingers deposited onto it for improved low-temperature performance), we can assign the measured PMT QE to the photo-cathode without major error. Low-temperature QEs average 30\% for xenon scintillation for the ZEPLIN-III phototubes [20].

\subsection{Electric fields}

The flat, disk-shaped LXe target ensures uniform electric fields (and light collection) above the PMT array. Inside this central ('fiducial') volume, high discrimination efficiency should be achieved. Interactions in outlying regions, which can probe non-vertical fringe fields, may still be corrected (or rejected) by use of three-dimensional event information. To ensure that this procedure is well understood, the simulation uses detailed electric field models for both phases. This is particularly important for the electroluminescence signal: the field determines not only how much ionisation is extracted from the interaction site, but also its drift time to the surface, the emission probability and location, the light yield in the gas and the temporal development of the signal.

Two-dimensional electric field maps are used by the electroluminescence model; by exploiting the cylindrical symmetry of the target, these maps can be kept small and are easily navigated. The standard GEANT4 tracking kernel is applied to all other particles, so there is no penalty to other processes. The ANSYS [21] finite-element software was used to produce field maps for several gas gaps, for a reference voltage of $1 \mathrm{~V}$ between the anode mirror and the cathode grid. The reverse-field region below the cathode grid, which suppresses S2 signals from low-energy PMT photons, is not implemented. Dielectric constants for the gas and liquid phases are $\epsilon_{\mathrm{g}}=1$ and $\epsilon_{1}=2$, respectively. The total interelectrode voltage (set interactively) scales the chosen field map to obtain the correct electric field strength. Fig. 2 (left) represents the ANSYS contour plot and the optimised meshing for the default map ( $5 \mathrm{~mm}$ gas). Electron trajectories obtained with a simple field navigation are shown in Fig. 2 (right). Note that the field is constant above the array, well away from the edge.

\subsection{The primary signal}

As a scintillator LXe compares favourably with the best crystals - although the exact light yield remains somewhat uncertain, with different studies indicating values in the range $\sim 30-80$ photons per $\mathrm{keV}$ of energy deposited (electron equivalent, hereafter ' $k e V e e ')$ - see $[22,23]$ and references therein. Here, we adopt a nominal figure of 60 photons/keVee (16.7 eV per scintillation photon). Correlation between VUV yield and electron lifetime is observed in ZEPLIN-II, where such a high photon yield is achieved and possibly surpassed. The LXe VUV luminescence is produced by the decay of singlet and triplet states of the $\mathrm{Xe}_{2}^{*}$ excimer. These can be excited directly by the interacting particle or as a result of recombination along the particle track $\left(\mathrm{Xe}_{2}^{+}+\mathrm{e}^{-} \rightarrow \mathrm{Xe}_{2}^{*}\right)$ [24,25]. Recombination is very efficient for nuclear recoil interactions, and so their scintillation is faster $(\tau \simeq 21 \mathrm{~ns}$ in a single-exponential approximation) than for electrons ( $\tau \lesssim 45 \mathrm{~ns}$, depending on energy) [24,26]. This discrimination technique was exploited in ZEPLIN-I [27].

The scintillation efficiency for nuclear recoils is lower than for electron interactions of the same energy. A zerofield quenching factor $\mathrm{QF}=0.22[26]$ was used in the simulation regardless of energy. Very recent data agree with this value at a few tens of $\mathrm{keV}$ (nuclear-recoil energy, ' $\mathrm{keVnr}$ '), but the trend at lower energies is still somewhat uncertain $[28,29]$. 

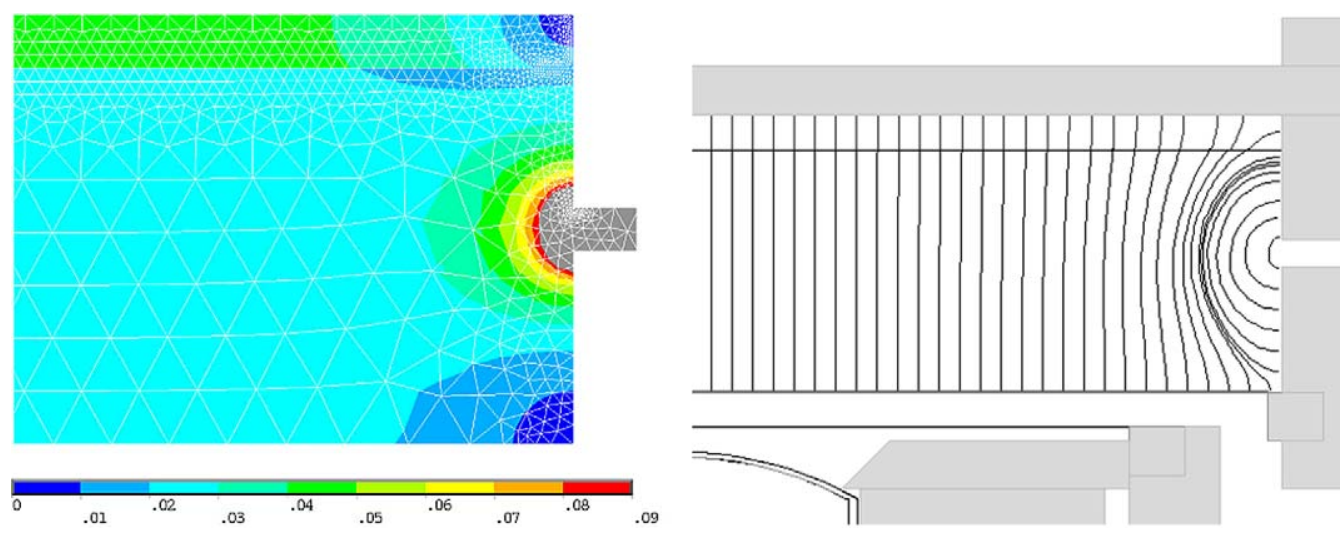

Fig. 2. Left: ANSYS [21] electric field contour map and optimised meshing in peripheral regions of the detector. Right: Simulated trajectories for charge released at the cathode grid and side electrode.

An external electric field suppresses recombination to a varying degree, affecting mainly the light yield and decay times for electron recoils, as depicted in Fig. 3. The scintillation model (adapted from that implemented in GEANT4) assumes a uniform electric field across both phases, i.e., yields and time constants are position-independent. This is not unreasonable considering that, for the range of interesting fields, these properties do not vary appreciably. The default yields for electron and nuclear recoils were set at 18 photons $/ \mathrm{keV}$ and 12 photons $/ \mathrm{keV}$, respectively, at the nominal $8 \mathrm{kV} / \mathrm{cm}$ field. The number of VUV photons is Gaussian-distributed (except when fewer than 10, in which case Poisson deviates are used), with unity Fano factor note that for relatively small S1 the overall energy resolution is instead dominated by photoelectron statistics.

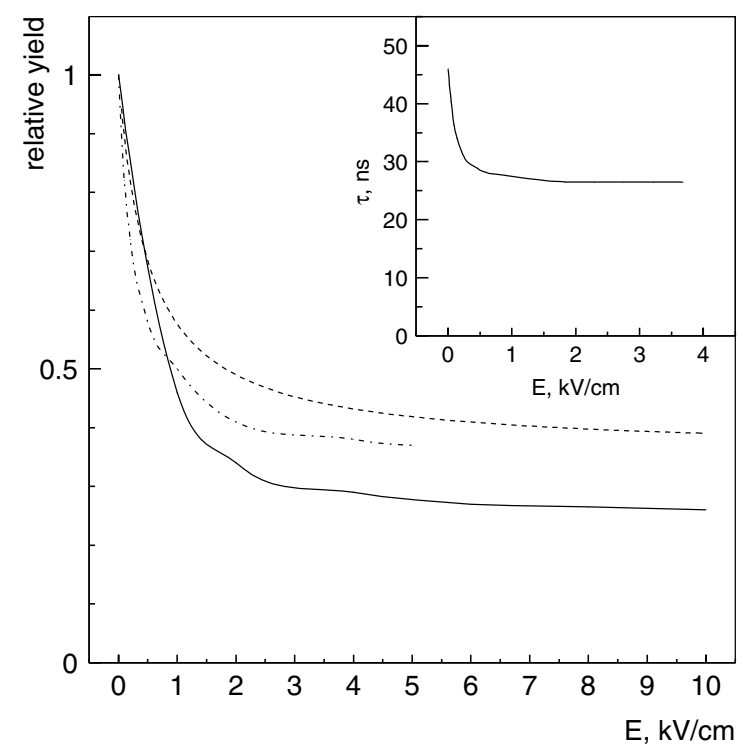

Fig. 3. Electric-field dependence of the scintillation yield for $1 \mathrm{MeV}$ electrons [24] (continuous line), $200 \mathrm{keV}$ Compton electrons [30] (dashed) and $122 \mathrm{keV} \gamma$-rays [29] (dash-dot). The inset shows the decay time constant (pulse area/amplitude) for $\sim 200 \mathrm{keV}$ Compton electrons [30].
Although pulse-shape analysis of S1 cannot provide as good a discrimination as in a zero-field detector, this may still prove a valuable diagnostic technique for calibration runs. For this reason, some timing properties were implemented in the scintillation model: a single-exponential decay is assumed, with time constants of $26 \mathrm{~ns}$ for electrons and $16 \mathrm{~ns}$ for recoils, as observed in our earlier work [7].

It should be pointed out that the scintillation and ionisation signals are correlated event-by-event, since recombination will contribute to either one or the other [31]. This correlation has not been taken into account in this model.

\subsection{The secondary signal}

Under the strong electric field, ionisation electrons are extracted from the interaction site and drifted upward to the liquid surface; once emitted into the gas phase they acquire enough energy to generate many VUV photons by electroluminescence. The S2 signal is proportional to the number of charge carriers extracted from the liquid, as well as the electric field and path length in the gas.

The number of electrons escaping recombination near the interaction site in LXe is calculated according to the electric field strength, the type of interacting particle and its energy. An energy $E_{\mathrm{d}}$ deposited in the liquid creates $E_{\mathrm{d}} / W$ free carriers, where $W$ is the mean energy required to create an electron-ion pair at infinite field. For $\gamma$-like interactions, we adopted $W_{\mathrm{e}}=15.6 \mathrm{eV}$ [32]. Some of the ionisation produced will recombine at finite field - this fraction depending on the $\gamma$-ray energy. Note that our present understanding of charge recombination is not accurate enough to constitute a microscopic model; for this reason, the dependence on particle energy (as opposed to energy deposited in LXe) is the only link to experimental data. We define a fractional charge yield, $N_{\mathrm{e}}$, representing the number of carriers that escape recombination, as

$\frac{1}{N_{\mathrm{e}}}=\left(\frac{30}{E_{\gamma}}+0.4\right) \frac{1}{E}+1.0$ 

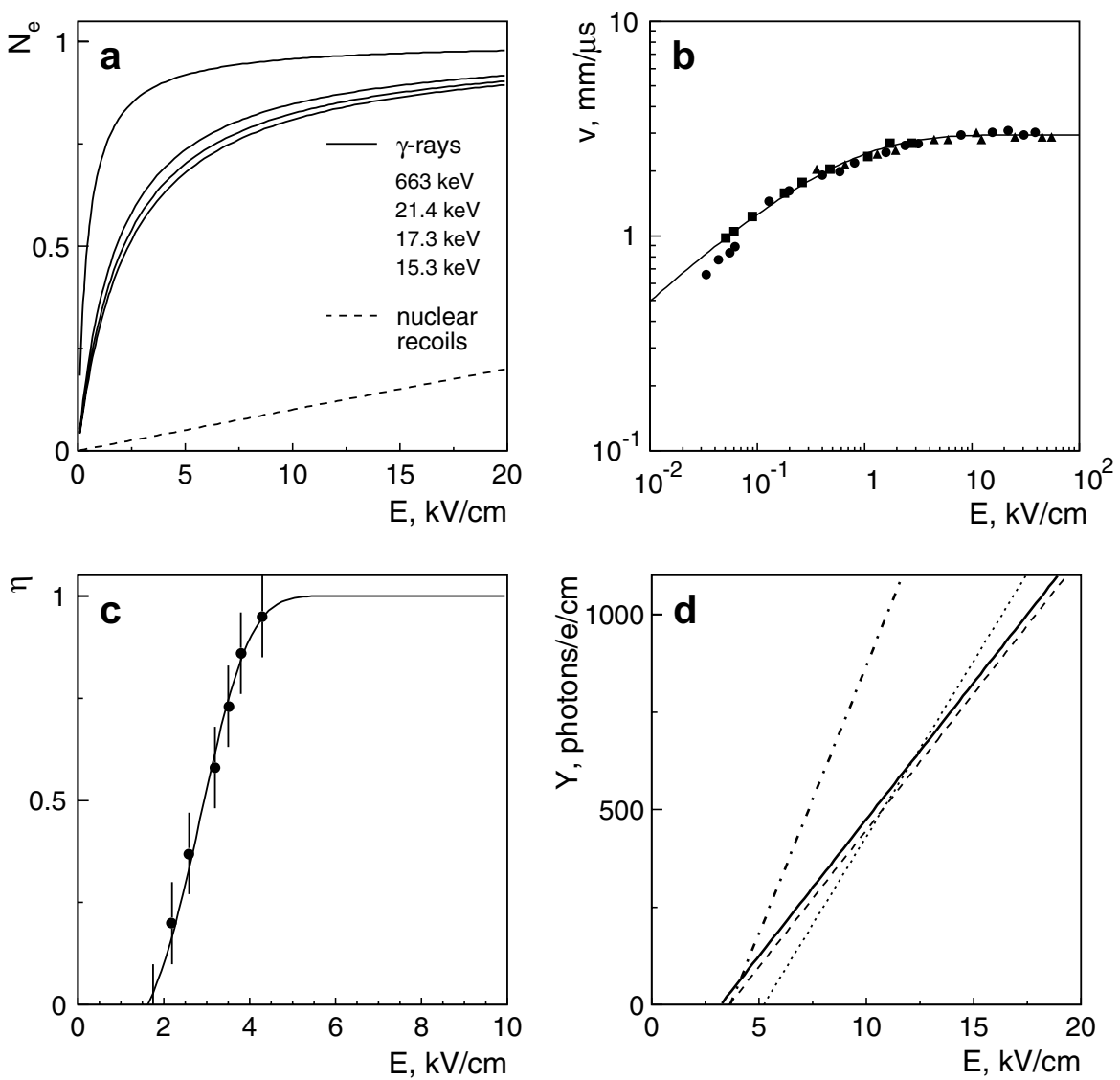

Fig. 4. Electric-field dependence of S2 parameters: (a) Relative ionisation yield adopted for $\gamma$-rays and nuclear recoils in LXe; $\gamma$-ray curves are parameterised from experimental data from Ref. [33] (ordered as in legend). (b) Drift speed in LXe, parameterised from data found in Ref. [35]. (c) Electron emission probability at liquid/gas interface, parameterised from experimental data in Ref. [37]. (d) Electroluminescence yields for gaseous xenon at 4 bar equivalent pressure at $0{ }^{\circ} \mathrm{C}$; continuous line: $Y=70 E-56 P_{\text {eq }}$ [39]; dashed line: $Y=70 E-63 P_{\text {eq }}[40]$; dotted line: $Y=90 E-117 P_{\text {eq }}[41]$; dashdotted line: saturated vapour, $Y=137 E-125 P_{\text {eq }}[42]$.

where $E_{\gamma}$ is the $\gamma$-ray energy in $\mathrm{keV}$ and $E$ is the field strength in $\mathrm{kV} / \mathrm{cm}[11]$. This function parameterises experimental data found in Ref. [33] and is plotted in Fig. 4a) for the photon energies considered in that study.

The ionisation yield for nuclear recoils was unknown at the start of this work, but it was thought to be much smaller than that for electron recoils. It was reasoned that, to first approximation, the number of ionisations and that of excitations producing scintillation would be suppressed by a similar amount, i.e., $W_{\mathrm{n}}=W_{\mathrm{e}} / \mathrm{QF} \simeq 71 \mathrm{eV}$. Under a finite electric field, only a fraction of this ionisation can be extracted from the nuclear track. Establishing a parallel with $\alpha$-particle ionisation yields, we adopted a linear dependence with field giving $10 \%$ charge yield at $10 \mathrm{kV} /$ $\mathrm{cm}$. Combining these two figures, we predicted that ZEPLIN-III should produce some 40 electrons from a $30 \mathrm{keV}$ nuclear recoil $\left(1.3 \mathrm{e}^{-} / \mathrm{keVnr}\right)$. Recent measurements suggest that nuclear-recoil ionisation may not be a linear function of either recoil energy or electric field, and could be 3-4 times higher than anticipated at $30 \mathrm{keVnr}$ at half the maximum ZEPLIN III field [34]. In view of this, some simulations were repeated with a yield four times higher than the default value $\left(5.2 \mathrm{e}^{-} / \mathrm{keVnr}\right)$, but still proportional to recoil energy and field.

The charge carriers released from each interaction site are tracked in the electric field until reaching either the liquid surface or a side-electrode. The drift speed in the liquid has been parameterised from experimental data [35], as shown in Fig. 4b). The high-field saturation value is $\simeq 3 \mathrm{~mm} / \mu \mathrm{s}$. Neither charge trapping by impurities nor carrier diffusion are considered at this stage in the simulation. For a diffusivity $D \sim 50 \mathrm{~cm}^{2} / \mathrm{s}[36]$, one can estimate that the charge arrival times at the liquid surface will be smeared by $0.1 \mu$ s following a $10 \mu$ s drift. Although this effect is not negligible when compared to the width of S2 itself, it can be taken into account at a later stage.

Upon reaching the surface, the normal component of the electric field in the liquid determines the fraction $\eta$ of charge emitted into the gas phase. Experimental data [37] has been parameterised as shown in Fig. 4c. The ionisation is then tracked in the gas field, generating electroluminescence photons along the way. A reduced drift speed of $1.5 \mathrm{~mm} / \mu \mathrm{s} /(\mathrm{V} / \mathrm{cm} /$ Torr $)$ is considered, obtained by extrapolating data in Ref. [38] to high fields. 
The electroluminescence yield, defined as the number of VUV photons created per extracted carrier and per $\mathrm{cm}$ of track, is calculated according to $Y=70 E-56 P_{\mathrm{eq}}$, where $E$ is the field in $\mathrm{kV} / \mathrm{cm}$ and $P_{\text {eq }}$ is the equivalent pressure in bar for the same gas density at $0{ }^{\circ} \mathrm{C}$ [39]. For a vapour pressure of $2.5 \mathrm{bar}\left(P_{\mathrm{eq}}=4 \mathrm{bar}\right)$ and a nominal field of $17.8 \mathrm{kV} / \mathrm{cm}$, a single electron extracted can produce $\sim 500$ photons over a $5 \mathrm{~mm}$ gas layer. This and similar yield equations, also shown in Fig. 4d), have been found for roomtemperature gaseous xenon. Recently, photon yields measured with saturated xenon vapour in equilibrium with the liquid phase were shown to be higher than those observed in the warm gas (the latter being consistent with previous experiments) [42]. This brings the prospect of even higher gain in the $\mathrm{S} 2$ channel.

The energy resolution achievable in S2 is dominated by fluctuations of the number of ionisation electrons extracted from the track, with lesser contributions from the ensuing stages (emission into the gas, electroluminescence and photon detection). It has long been acknowledged that the intrinsic resolution achievable by charge readout in LXe is worse than both the calculated Fano factor $(F=0.04$ [36]) and the Poisson limit $(F=1)$ - see, e.g. [31]. This has been attributed to the (small-number) statistics of $\delta$ ray production acting together with charge recombination, which can persist even under electric fields as high as $20 \mathrm{kV} / \mathrm{cm}$ [43]. This effect is likely to be less marked for energies up to a few tens of keVee. Although most results presented here are for unity Fano factor, $F=0$ and $F=10$ were also considered. Further (Gaussian) deviates are applied at the photon generation stage to smear the number of photons produced per electron extracted.

\subsection{DAQ model}

In each event optical tracking ends with the probabilistic detection of photons arriving at each PMT photo-cathode. The phe creation times are then histogrammed into long timelines with $2 \mathrm{~ns}$ binning. Two such timelines of the sum of all 31 channels are shown in Fig. 5 for $10 \mathrm{keVee}$ electron and nuclear recoils $(15 \mathrm{keVnr})$ interacting $5 \mathrm{~mm}$ below the liquid surface. ${ }^{2}$ The two $\mathrm{S} 2$ signals differ by a factor of 10 in area for a similar $\mathrm{S} 1(\simeq 10$ phe, just visible at $2000 \mathrm{~ns})$.

To obtain the PMT output voltage, the impulse response function (i.e., single phe response) is calculated analytically [44] and numerically convolved with the phe timeline [45]. For a gain of $2 \times 10^{5}$, the mean single phe height is $0.16 \mathrm{mV}$ and the pulse area is $1.6 \mathrm{pVs}$. Such low gain should avoid saturation effects following very large S2 signals. Wideband amplifiers add further gain $(\times 50)$ and noise $(30 \mu \mathrm{Vrms}$ at input) to the signal. Finally, the voltage is digitised with 8 -bit resolution. The dual-range

\footnotetext{
${ }^{2}$ Note that keVee is defined as the equivalent (visible) energy for electrons taking into account the field-induced suppression of S1, so $\mathrm{keVee}=(\mathrm{QF} / 0.3) \mathrm{keVnr} \simeq 2 / 3 \mathrm{keVnr}$.
}

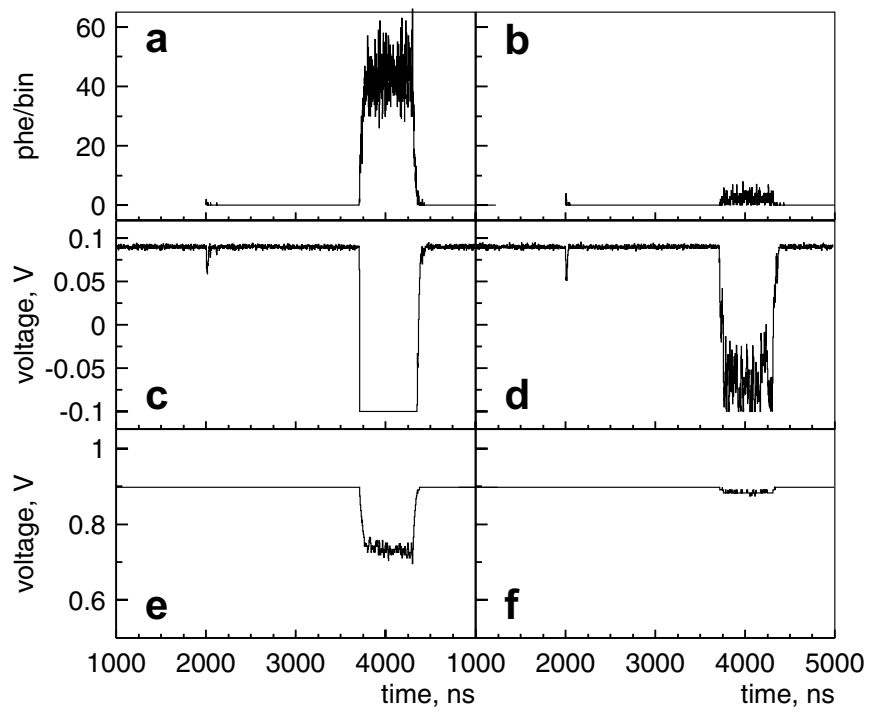

Fig. 5. Simulated timelines (31-channel sum) for $10 \mathrm{keVee}$ electron (left) and nuclear recoil interactions (right). (a) and (b) are photoelectron timelines; (c) and (d) show the high-sensitivity DAQ channel; (e) and (f) show the low-sensitivity DAQ channel.

DAQ records two traces per channel at $100 \times$ full-scale difference. Fig. 5(c)-(f) shows how the summed timeline would appear on both (note that noise from a single channel is considered).

\section{Simulation results}

\subsection{Simulations of optical response}

The optical sensitivity to S1 and S2 are key parameters which affect the detector performance at many levels. The primary scintillation yield across the chamber is shown in Fig. 6 (left) for the default parameters $(5 \mathrm{~mm}$ of gas, $34.6 \mathrm{~cm} \mathrm{LXe} \mathrm{absorption} \mathrm{length,} 15 \% \mathrm{Cu}$ reflectivity). It assumes a zero-field yield of 60 photons $/ \mathrm{keV}$ in both phases. The light collection is quite uniform across the active LXe region; a reference value for the centre of the target is $3.4 \mathrm{phe} / \mathrm{keV}$ at zero field $(1.0 \mathrm{phe} / \mathrm{keV}$ at maximum field for electron recoils).

Increasing the mirror reflectivity to $R=90 \%$ would improve the zero-field value to $4.2 \mathrm{phe} / \mathrm{keV}$. This relatively modest increase is due to the fact that most scintillation photons are internally reflected by the liquid/gas interface and not by the top electrode (and many that do reach the top plate are externally reflected at the liquid surface and remain trapped in the gas phase). Filling the detector to immerse the top mirror decreases the yield from 3.4 phe/ $\mathrm{keV}$ to $2.3 \mathrm{phe} / \mathrm{keV}$. Increasing the LXe absorption length to a more realistic $100 \mathrm{~cm}$ improves the reference yield to $4.0 \mathrm{phe} / \mathrm{keV}$ with low- $R$ mirrors.

The S2 light yield was simulated by generating a constant charge over a square grid located under the liquid surface; these electrons are emitted to the gas phase with a spatially-dependent efficiency where they generate a varying number of photons - thus taking into account 

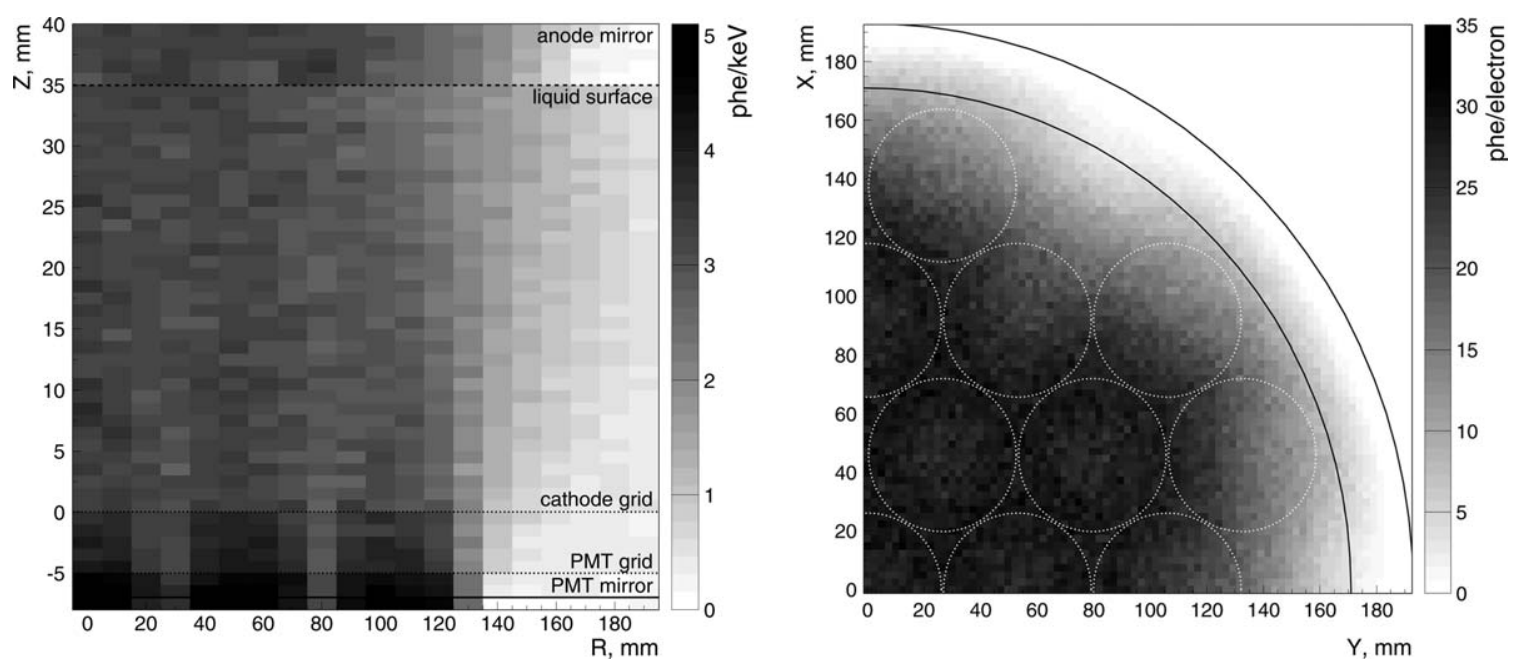

Fig. 6. Left: light yield from primary scintillation (S1) with no applied electric field. Right: light yield per ionisation electron generated in the liquid (surface emission probability combined with electroluminescence yield at nominal field).

the position-dependent electric field. The result is shown in Fig. 6 (right). The two concentric lines represent the cathode grid and active volume radii. The light collection is quite uniform across the central part of the chamber, where an average 26 phe are generated per electron extracted. Fig. 7 shows the S2 distribution for one and two electrons emitted into the gas (assuming unity extraction efficiency). Two populations can be clearly seen when outlying events are cut ( $>156 \mathrm{~mm}$ radius), confirming the sensitivity to single electrons extracted from the target.

\subsection{Position reconstruction}

In ZEPLIN-III a fiducial region can be identified in the target without physical boundaries by reconstructing the three coordinates of the interaction. Spatial sensitivity is

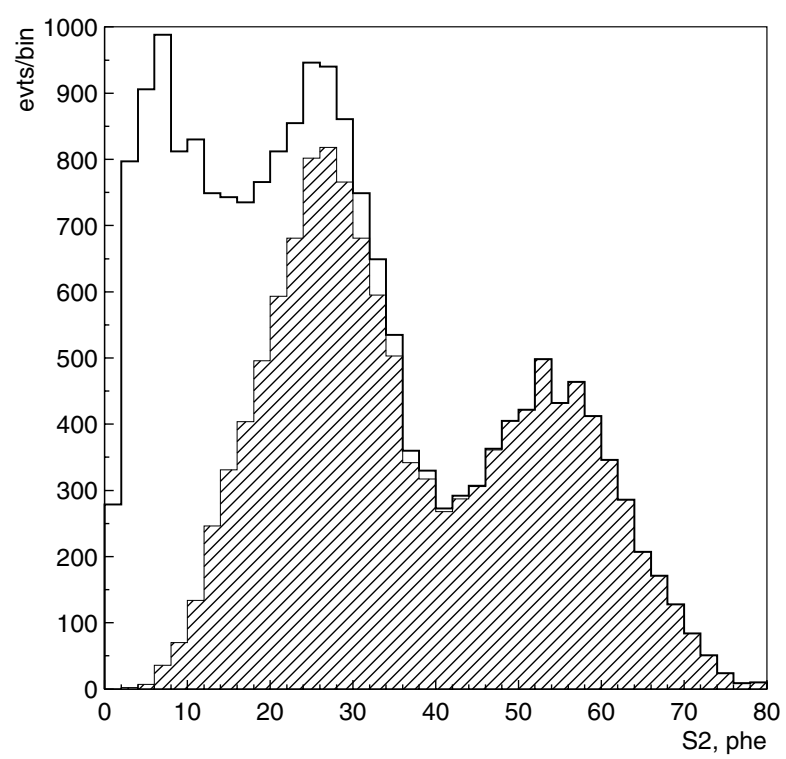

Fig. 7. S2 spectrum for one and two ionisation electrons extracted from the liquid assuming unity surface emission probability. The shaded distribution includes only events in the inner $8 \mathrm{~kg}(156 \mathrm{~mm})$. important in low-background detectors since surface contamination is a source of low-energy events (e.g., nuclear recoils from $\alpha$ decay). Interactions occurring in regions where the electric field and the light collection are not uniform should also be excluded.

In two-phase detectors the $z$ coordinate is obtained with sub-mm precision by the drift time in LXe, as given by the time separation between $\mathbf{S} 1$ and $\mathbf{S} 2$. The horizontal $(x, y)$ position is reconstructed using a template method; this is outlined here and described in detail elsewhere [46,11]. An analytical method was also shown to be viable [47].

A template was generated by full optical simulation of the S2 response produced from 20,000 locations in the gas phase, organised in a square mesh with $2.5 \mathrm{~mm}$ pitch. From each point $1.5 \times 10^{6}$ photons were released and tracked, and the signals produced in each PMT were stored to form a template with $20,000 \times 31$ entries. After simulation of a realistic event, $\mathrm{S} 2$ distributions are compared with the stored template responses, and the point with the lowest $\chi^{2}$ is retrieved as the best estimate of $(x, y)$.

An important requirement of the algorithm is that it should be able to run on-line during data acquisition. Although the event rate expected underground is only a few events/s, it will be higher during surface tests and calibration runs, easily reaching the hardware limit of 100 events/s. To improve the performance of the algorithm, only a sub-set of the template is searched in each event. This population should represent the entire template and must therefore have homogeneous space coverage. This was achieved by selecting the sub-set using a two-dimensional Sobol sequence - one of a family of so-called 'quasi-random' sequences designed to cover a given interval homogeneously [45]. A faster local search is then performed in the vicinity of the selected point. A 2500-point sub-set proved to be the limit where the resolution is not affected. This allows the algorithm to achieve a reconstruction frequency $>200 \mathrm{~Hz}$ (on a $3 \mathrm{GHz} \mathrm{CPU}$ ), which is significantly faster than the maximum DAQ rate. 


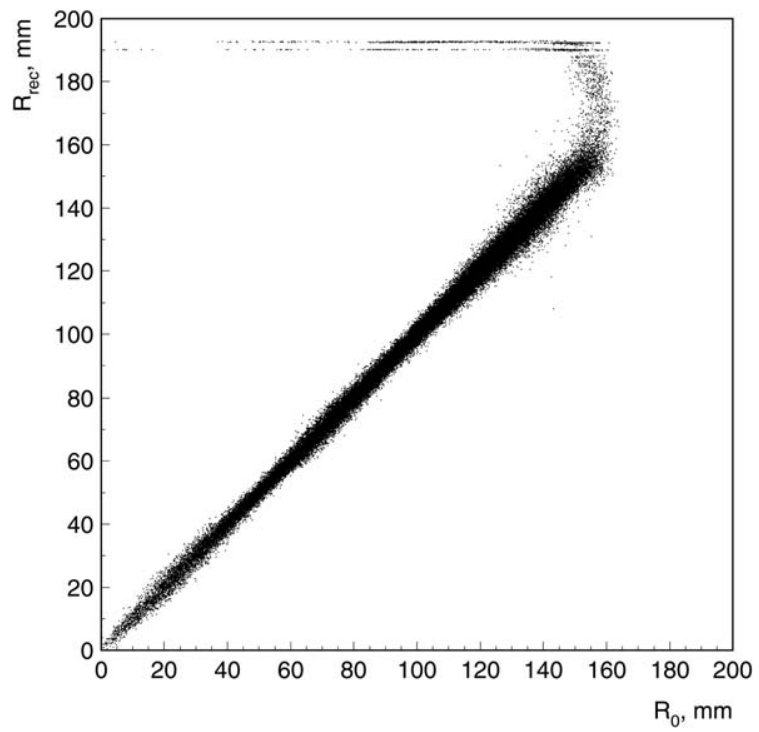

Fig. 8. Plot of the reconstructed radius $R_{\text {rec }}$ as a function of the real radius $R_{0}$ of the interaction for the whole detector for 10 electrons extracted from the liquid.

Random events with 1,4 and 10 ionisation electrons extracted from the liquid were simulated and their $(x, y)$ coordinates reconstructed, showing resolutions of $\simeq 10 \mathrm{~mm}$ (FWHM) for extremely small energy deposits (1 electron) and a few millimetres near the threshold energy (10 electrons). Fig. 8 shows the reconstructed radius for random interactions across the whole active region for 10 electrons extracted, subject to $\mathrm{S} 2>10$ phe (this cut eliminates fringe events with low $\mathrm{S} 2$ values due to poor light collection and weaker field).

Ultimately, the merit of the algorithm must be judged by the size and quality of the fiducial volume that can be derived. Outlying events, with distorted signals, can be placed nearer the centre by the routine and misinterpreted as interesting ones. Contamination from these events was studied for several test volumes, showing negligible crossboundary migration $\left(\sim 1: 10^{3}\right)$ for up to $8 \mathrm{~kg}(156 \mathrm{~mm}$ radius) down to very small energy deposits ( $\mathrm{S} 2>$ 100 phe). This is a significant improvement over the initial design goal of $5-6 \mathrm{~kg}$.

\subsection{Calibration}

Calibrating the S1 and S2 channels with electron recoils of suitably low energies throughout the detector volume is not a trivial task. With increasing target masses, calibration with reference to a single point or small region may prove inaccurate. S1 and S2 depend on local electric fields and optical properties which may differ between the LXe bulk and near electrodes and reflectors.

In ZEPLIN-III the $\gamma$ calibration strategy will consist of (i) a relatively low-energy calibration with ${ }^{57} \mathrm{Co} \gamma$-rays for light/charge yield measurements and long-term monitoring of the detector stability; (ii) calibration with high-energy

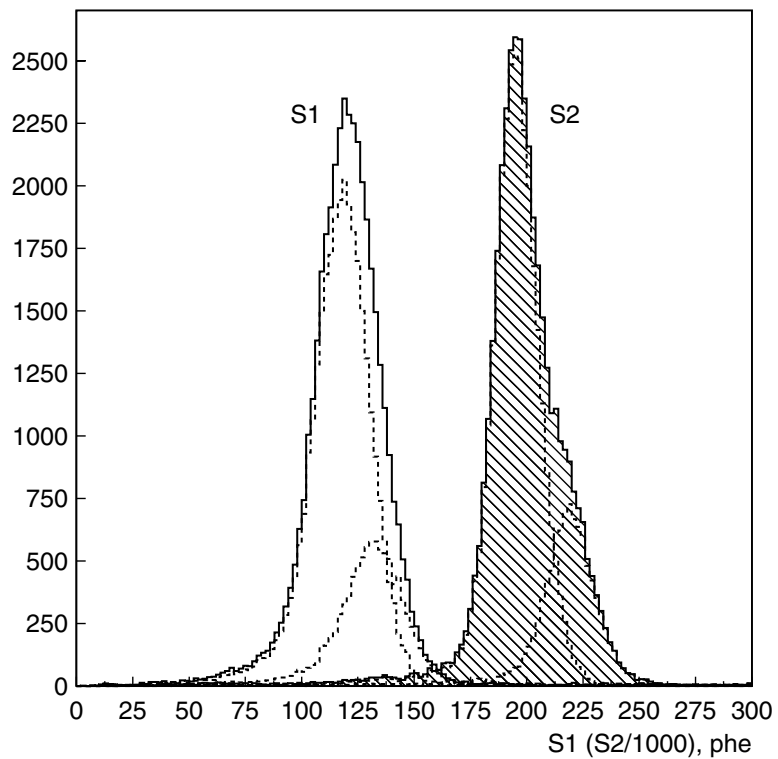

Fig. 9. S1 and S2 energy spectra in the inner $8 \mathrm{~kg}$ from collimated ${ }^{57} \mathrm{Co}$ source located above the detector. S2 (shaded) is scaled down by a factor of 1000 . The contribution of the individual $\gamma$ energies $(122.1 \mathrm{keV}$ and $136.5 \mathrm{keV}$ ) is also shown.

$\gamma$-rays from ${ }^{60} \mathrm{Co}$ in order to populate near-threshold energies with low-energy Compton electrons; (c) low-energy $\gamma$-rays from inelastic neutron scattering and from the radioactive decay of xenon and copper radioisotopes produced by neutron activation, which can be used to check the volume uniformity (e.g., inelastic scattering of neutrons emitted by the PMTs cause one such feature seen at $40 \mathrm{keV}$ in Fig. 13 in the following section).

We outline here the simulation results for a collimated ${ }^{57} \mathrm{Co}$ source placed above the vacuum vessel, with reference to Fig. 9. In spite of over $1 \mathrm{~cm}$ of intervening copper, a reasonable number of $122.1 \mathrm{keV}$ and $136.5 \mathrm{keV}$ photons get through to the target $(14 \mathrm{keV} \gamma$-rays are completely absorbed). Low-intensity $(<1 \%){ }^{57} \mathrm{Co}$ lines at higher energies provide additional calibration points, most notably the $692 \mathrm{keV} \gamma$-ray (not shown). The S1 energy resolution for the $122 \mathrm{keV}$ line is $25 \%$ (FWHM), close to the value expected from phe statistics. The energy resolution in S2 is slightly better, being determined mainly by the Fano factor at these energies: $\mathrm{FWHM} \simeq 12 \%$ for $F=1$. The limiting cases are $9 \%(F=0)$ and $15 \%(F=10)$ - comparable to the $11 \%$ energy separation between the two lines.

Calibration of the response to nuclear recoils will rely on irradiation with an $\mathrm{Am}-\mathrm{Be}(\alpha, \mathrm{n})$ source in conjunction with independent measurements of the scintillation QF to establish the energy scale. To calculate the recoil spectrum and the required exposure, a $0.1 \mathrm{GBq} \mathrm{Am}-\mathrm{Be}$ source placed above the outer vessel was simulated. The input neutron spectrum used was that reported in Ref. [48], with no consideration given to $\gamma$-rays from the source. The resulting spectrum for single elastic scatters is similar in shape to that obtained for background neutrons from the PMTs which is shown in Fig. 13. Some $\sim 50,000$ events/h 
are expected above $10 \mathrm{keVnr}$ in the $8 \mathrm{~kg}$ fiducial volume, with single scatters constituting approximately $60 \%$ of all recoil events.

Activation of xenon and other materials has also been studied and found not to constitute a problem except for unreasonably large exposures. Low energy signals are expected from ${ }^{125} \mathrm{I} \mathrm{X}$ - and $\gamma$-rays (EC, $T_{1 / 2}=59.4$ days), which follows from neutron capture on ${ }^{124} \mathrm{Xe}$. Other Xe isotopes and their metastable states contribute low-energy $\gamma$-rays which persist for several days after irradiation, namely ${ }^{129} \mathrm{Xe},{ }^{131} \mathrm{Xe}$ and ${ }^{133} \mathrm{Xe}$. Neutron capture on copper is important but mainly during the exposure, since the products are short-lived. Small amounts of ${ }^{55} \mathrm{Fe}$ will be created at the electrode grids, but these low-energy photons can be cut from the data by drift-time.

\subsection{Discrimination power}

Low-energy electrons and Xe nuclei $\left(5 \times 10^{5}\right)$ were generated randomly throughout the active volume with a constant energy spectrum. Datasets were produced with (a) ionisation Fano factors $F=0,1$ and 10 and (b) nuclearrecoil ionisation yields proportional to both electric field strength and recoil energy generating an average 12.5 and 50 electrons at $10 \mathrm{keVnr}$ (the two scenarios described in Section 2.5). The number of S1 and S2 phe in each PMT was obtained with full optical tracking. The plot in Fig. 10 shows the ratio S2/S1 for the electron- and nuclear-recoil populations as a function of energy $(\propto \mathrm{S} 1)$ for $F=1$ and the lower ionisation yield. Interactions outside the $8 \mathrm{~kg}$ fiducial region were rejected.

The lines along the tail of the upper population mark $\gamma$ rejection efficiencies of $10^{n}: 1$ - indicating the $\mathrm{S} 2 / \mathrm{S} 1$ ratio at which one event leaks to the nuclear recoil population for every $10^{n}$ electron recoils. Higher rejection efficiency boundaries could not be calculated since they would require an unreasonably long simulation. Fig. 11 plots the recoil detection efficiencies obtained with this procedure. In conclusion, $10^{4}: 1$ discrimination to $\gamma$-rays can be reached between $8 \mathrm{keVnr}$ and $14 \mathrm{keVnr}$ for $F=1$. The combination of high ionisation yield and $F=10$ (not shown) produces a less competitive result.

One particular class of problematic event requires consideration: $\gamma$-rays hitting both the active region and the 'dead' volume below the cathode will produce lower S2/ $S 1$ ratios than single interactions in the active volume, since individual S1 signals will add up but only one $\mathrm{S} 2$ is generated. The discrimination efficiency will therefore be lower for these events. Simulation of background $\gamma$-rays emitted by the PMTs (the dominant source of electron recoils) indicates $\sim 2700$ of these multiple scatters per day. The vast majority can be rejected by restricting the S2 location to the fiducial volume $(<156 \mathrm{~mm}$ radius) and requiring that the energy deposits in either regions be below $10 \mathrm{keVee}$ (at higher energies they can easily be discriminated). Ten or so events per day survive these cuts. Of these, the most dangerous ones deposit a small energy in the target (small

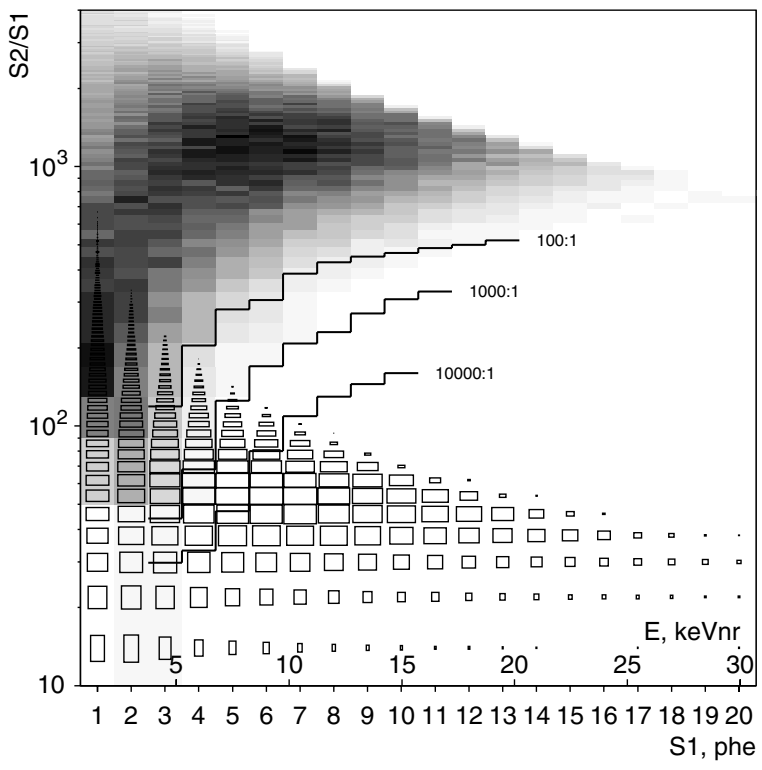

Fig. 10. S2/S1 distributions for electrons (upper population) and nuclear recoils (lower population) in the inner $8 \mathrm{~kg}$, with lower ionisation yield and unity Fano factor. The thick lines represent the boundaries for a given $\gamma$ ray discrimination efficiency. The energy axis considers $\mathrm{QF}=0.2$.

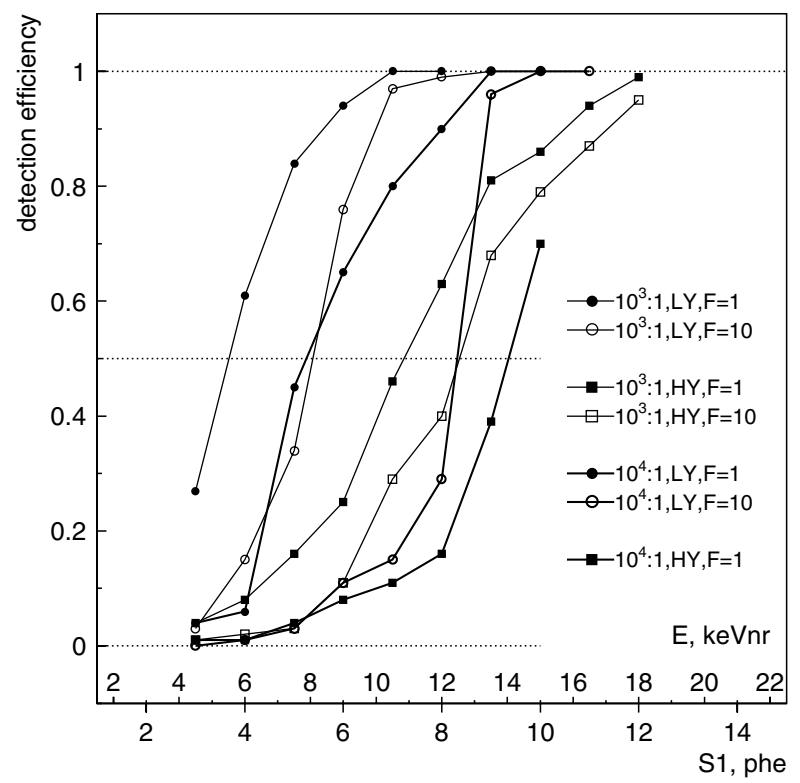

Fig. 11. Nuclear recoil detection efficiency curves for $10^{3}: 1$ and $10^{4}: 1 \gamma$ discrimination for lower (LY, thin lines) and higher (HY, thick lines) ionisation yields.

S2) and a comparatively large energy in the dead region (large S1). Many such cases can be identified by analysing the S1 spatial distribution across the array: a more localised interaction (with many phe in a single channel) is expected relative to a similar amplitude $\mathrm{S} 1$ in the active region. This analysis can be extended to take into account that the S1 location should be consistent with the $(x, y)$ point reconstructed from S2. Very few events per day are expected to escape these cuts, and a modest discrimination 
efficiency $(10 \times$ lower than that achieved in the fiducial region) should reduce those to an insignificant contribution.

\subsection{Detector sensitivity}

The performance of ZEPLIN-III as a dark matter detector relies ultimately on the number of background events producing signatures which cannot be distinguished from those of WIMPs. Neutrons are the obvious irreducible source of background, but electron recoils which evade discrimination must also be considered. In addition, electron recoils dominate the detector trigger rate, and this has implications for the DAQ operation. In this section, we calculate the two types of background from dominant internal and external sources by tracking background particles and looking for energy deposits in the target. Full optical tracking is not performed since the very large dynamic range of the background signals would make this computation overwhelming.

\subsubsection{Electron-recoil backgrounds}

Trace radioactivity in the PMTs is the major source of background of both $\gamma$-rays and neutrons - and consequently the rate of interactions and total energy (light) deposited in active region. The PMT contamination levels were measured at $250 \mathrm{ppb}$ in $\mathrm{U}, 290 \mathrm{ppb}$ in Th and $1350 \mathrm{ppm}$ in K. $\gamma$-rays from ${ }^{40} \mathrm{~K}$ plus the secular equilibrium spectra for the ${ }^{238} \mathrm{U}$ and ${ }^{232} \mathrm{Th}$ chains [49] were generated uniformly from the PMT glass and fully tracked until they were absorbed or left the outer vacuum vessel (although the radioactivity is concentrated mainly at the graded seal near the PMT windows and the glass bases, this approximation is not unreasonable). Note that GEANT4 can generate the $\gamma$ spectrum for the full $\mathrm{U}$ and Th chains, in good agreement to the one used here, and producing in addition the correct average number of $\alpha, \beta$ and $\gamma$-rays per parent decay. Low-energy $\mathrm{X}$-rays, $\alpha$ and $\beta$ radiation interacting very close to the PMT bodies are unlikely to trigger more than one PMT due to the copper screens in which they are located, and for this reason were not considered.

The differential energy spectrum in the target is depicted in Fig. 12 (traces A and B). We predict a low-energy rate of $10 \mathrm{dru}$ ( $1 \mathrm{dru}=1 \mathrm{event} / \mathrm{kg} / \mathrm{day} / \mathrm{keVee})$, confirming earlier results [11]. An interaction rate of $5 \mathrm{~Bq}$ will be caused above the PMT windows ( $\mathrm{S} 1$ trigger rate), decreasing to $2.5 \mathrm{~Bq}$ above the cathode grid ( $\mathrm{S} 2$ trigger rate). This corresponds to an average rate of energy deposited just under $2 \mathrm{MeV} / \mathrm{s}$.

A second internal source of electron recoils is the $\beta$ decay of ${ }^{85} \mathrm{Kr}\left(T_{1 / 2}=10\right.$ year $)$ contaminating the Xe itself. Extracted from the atmosphere in the mid-1960s, the ZEPLIN-III xenon has a low ${ }^{85} \mathrm{Kr}$ content, equivalent to $\sim 5 \mathrm{ppb} \mathrm{Kr}$. The (Coulomb-corrected) $\beta^{-}$energy spectrum generated by GEANT4 is tracked in the target, leading to $0.1 \mathrm{dru}$ at low energies (trace D in Fig. 12).

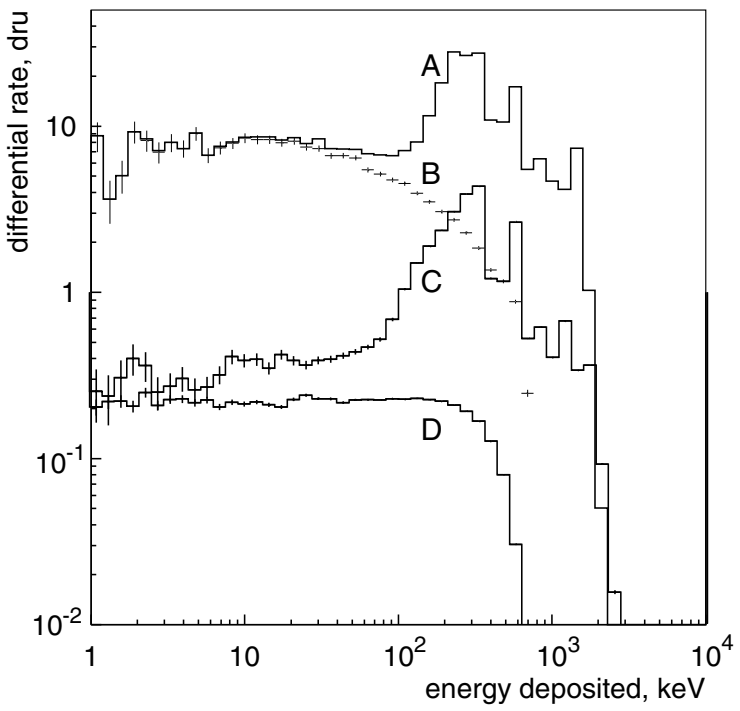

Fig. 12. Electron-recoil background rates: A - total energy deposit in the active region from PMT $\gamma$-rays; $\mathrm{B}$ - same as $\mathrm{A}$, but for single interactions; $\mathrm{C}-\gamma$-rays from $10 \mathrm{~Bq} / \mathrm{m}^{3}$ of ${ }^{222} \mathrm{Rn}$ progeny $\left({ }^{214} \mathrm{Bi}+{ }^{214} \mathrm{~Pb}\right)$ decaying in air inside the lead shielding; $\mathrm{D}$ - background from $\beta^{-}$decay of ${ }^{85} \mathrm{Kr}(5 \mathrm{ppb}$ $\mathrm{Kr}$ ) in the active volume.

The presence of ${ }^{222} \mathrm{Rn}$ and its progeny in the air surrounding the detector is a source of radioactive background - mainly due to the short-lived $\gamma$-emitters ${ }^{214} \mathrm{Bi}$ and ${ }^{214} \mathrm{~Pb}$. In Ref. [50] an upper limit is placed on the differential rate expected from these isotopes in a large Xe detector surrounded by air contaminated with $10 \mathrm{~Bq} / \mathrm{m}^{3}$ in ${ }^{222} \mathrm{Rn}$; the result in reproduced in Fig. 12C. We note that the actual contribution from these isotopes is likely to be even smaller, as both the air volume and contamination level considered are too generous.

Finally, the cavern walls (mainly $\mathrm{NaCl}$ ) emit $\gamma$-rays, requiring extensive lead shielding around the detector. A calculation based on radioactivity measurements of the Boulby rock (67 ppb U, 127 ppb Th, 1300 ppm K) suggests that a lead castle $15 \mathrm{~cm}$ thick can attenuate the $\gamma$ background to $<0.01 \mathrm{dru}$ [51].

In conclusion, a total electron-recoil background of $10 \mathrm{dru}$ is expected in a shielded detector, dominated by PMT $\gamma$-rays.

\subsubsection{Nuclear-recoil backgrounds}

Energetic neutrons are produced due to radioactive contamination of detector components and its surroundings with the uranium and thorium decay chains, by spontaneous fission (mainly of ${ }^{238} \mathrm{U}$ ) and the $(\alpha, \mathrm{n})$ reaction. Cosmicray muons also generate neutrons in spallation reactions and secondary cascades.

Operation deep underground attenuates the muon flux by several decades, but a few neutrons are still produced by muons interacting in the detector materials and the cavern rock. Muon-induced neutron production at the Boulby Underground Laboratory ( 2800 mwe) has been studied in the context of a large-scale Xe detector in Refs. [52-54]. For the shielding configuration anticipated for 


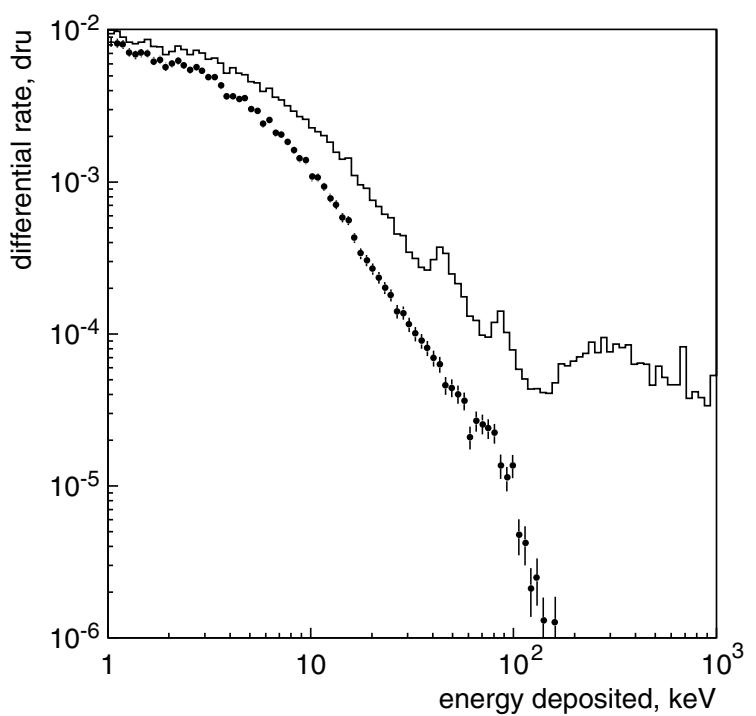

Fig. 13. Differential energy spectrum (evt $/ \mathrm{kg} / \mathrm{day} / \mathrm{keVee}$ ) from PMT neutrons. The line represents total energy deposited in the target (electron and nuclear recoils), whilst the markers show single nuclear recoils only (in the latter case, the abscissa corresponds to $\mathrm{keVnr}$ ).

ZEPLIN-III one can expect $\lesssim 1$ single nuclear recoil event per year above $10 \mathrm{keVnr}$ in a $8 \mathrm{~kg}$ fiducial volume.

The neutron flux in the cavern is instead dominated by $\mathrm{U} / \mathrm{Th}$ radioactivity in the Boulby rock. The flux at the rock face has been simulated at $\approx 2 \times 10^{-6} \mathrm{n} / \mathrm{s} / \mathrm{cm}^{2}$ above $1 \mathrm{MeV}$ (excluding backscattering of reentering neutrons) for the above rock contamination [54,52]. This flux would easily overwhelm an unshielded detector. Hydrocarbon shielding $\left(20-30 \mathrm{~g} / \mathrm{cm}^{2}\right)$ located inside the lead castle should attenuate the neutron flux to acceptable levels - producing fewer than $\sim 1$ nuclear recoil per year for a complete $(4 \pi)$ shield.

Neutrons produced inside the detector shielding can only be avoided by construction with low-background materials and the use of an active veto surrounding the detector, to record neutrons in coincidence with the target. Construction from mainly high-purity copper (U/Th levels $<1 \mathrm{ppb})$ and the relatively high threshold for $(\alpha, \mathrm{n})$ in $\mathrm{Cu}$ ensure a very low neutron background from the vicinity of the target from most components, with the notable exception of the PMTs.

Neutron spectra from fission and $(\alpha, n)$ reactions in the PMT borosilicate glass were calculated using the
SOURCES-4A code [55] modified in the way described in Ref. [52], for the U/Th contamination levels mentioned previously. Fig. 13 shows the differential energy spectrum caused in the target by PMT neutrons emitted isotropically and uniformly from the PMT glass. The figure represents the total energy deposited (elastic plus inelastic events) in addition to single nuclear-recoil events. PMT neutrons reach $\sim 10^{-3}$ dru (e.e.) at low energies, typically contributing 25 events/year above $10 \mathrm{keVnr}$ in the $8 \mathrm{~kg}$ fiducial mass.

\subsubsection{WIMP sensitivity}

Background from the 31 PMTs dominates in a shielded ZEPLIN-III by a large margin. Some $20-40$ events/year will be due to neutrons (depending on the energy acceptance considered). In addition, although most $\gamma$-rays can be removed by S2/S1 discrimination, some will 'leak' into the nuclear recoil population for any realistic discrimination cut adopted. Increasing the latter (e.g., from $10^{3}: 1$ to $10^{4}: 1$ ) also raises the energy threshold, as illustrated in Fig. 11 (fortunately, it also decreases the number of neutron events).

The yearly neutron and $\gamma$ rates are presented in Table 1 for the ionisation yields mentioned previously $\left(1.3\right.$ and $5.2 \mathrm{e}^{-} /$ keVnr) and $F=1$. We adopt a $50 \%$ energy threshold, $E_{0}$, from the efficiency curves in Fig. 11. The neutron distribution (Fig. 13) is integrated above $E_{0}$. The number of $\gamma$-rays is obtained by integrating a constant rate of 10 dru (Fig. 12) over 2 keVee (very few non-discriminated $\gamma$-rays should remain above $E_{0}+2 \mathrm{keVee}$ ). The sensitivity limits for the WIMP-nucleon cross-section (spin-independent interaction) are also shown for the minimum of the sensitivity curve (60 GeV WIMP mass), for an exposure of $240 \mathrm{~kg} \times$ days ( 1 month at $100 \%$ duty cycle) and $3000 \mathrm{~kg} \times$ days ( 1 year). These were calculated as described in Ref. [56].

A limiting sensitivity of $3-4 \times 10^{-8} \mathrm{pb}$ can be achieved even in a high ionisation yield scenario. This is due to the fact that the reduced energy acceptance for nuclear recoils affects the neutron background more than the WIMPinduced spectrum at these energies. This sensitivity is similar to the original design prediction, and confirms that ZEPLIN-III can probe deep into the parameter space favoured by SUSY in its original design.

An active veto system installed around the detector can be used to reject events in coincidence with the target. A

ZEPLIN III background rates and WIMP sensitivity

\begin{tabular}{|c|c|c|c|c|c|c|c|c|c|c|}
\hline & \multicolumn{5}{|c|}{ Low recoil ionisation yield } & \multicolumn{5}{|c|}{ High recoil ionisation yield } \\
\hline & \multirow{2}{*}{$\begin{array}{l}E_{0} \\
\mathrm{keVnr}\end{array}$} & \multicolumn{2}{|c|}{ event/year } & \multicolumn{2}{|l|}{$\sigma_{\min }, \mathrm{pb}$} & \multirow{2}{*}{$\begin{array}{l}E_{0} \\
\mathrm{keVnr}\end{array}$} & \multicolumn{2}{|c|}{ event/year } & \multicolumn{2}{|l|}{$\sigma_{\min }, \mathrm{pb}$} \\
\hline & & $\mathrm{n}$ & $\gamma$ & $240 \mathrm{~kg} \times$ days & $3000 \mathrm{~kg} \times$ days & & $\mathrm{n}$ & $\gamma$ & $240 \mathrm{~kg} \times$ days & $3000 \mathrm{~kg} \times$ days \\
\hline $\begin{array}{l}10^{3}: 1 \gamma \text { disc. } \\
10^{4}: 1 \gamma \text { disc. }\end{array}$ & $\begin{array}{l}5.5 \\
8\end{array}$ & $\begin{array}{l}40 \\
31\end{array}$ & $\begin{array}{r}60 \\
6\end{array}$ & $\begin{array}{l}1.0 \times 10^{-7} \\
6.9 \times 10^{-8}\end{array}$ & $\begin{array}{l}6.7 \times 10^{-8} \\
3.4 \times 10^{-8}\end{array}$ & $\begin{array}{l}11 \\
14\end{array}$ & $\begin{array}{l}24 \\
16\end{array}$ & $\begin{array}{r}60 \\
6\end{array}$ & $\begin{array}{l}1.5 \times 10^{-7} \\
8.8 \times 10^{-8}\end{array}$ & $\begin{array}{l}9.5 \times 10^{-8} \\
3.6 \times 10^{-8}\end{array}$ \\
\hline $\begin{array}{l}\text { Veto installed }^{\mathrm{a}} \\
\text { PMT upgrade }\end{array}$ & $\begin{array}{l}8 \\
8\end{array}$ & $\begin{array}{l}16 \\
1.6\end{array}$ & $\begin{array}{l}2 \\
0.2\end{array}$ & $\begin{array}{l}4.7 \times 10^{-8} \\
2.2 \times 10^{-8}\end{array}$ & $\begin{array}{l}1.8 \times 10^{-8} \\
4.2 \times 10^{-9}\end{array}$ & $\begin{array}{l}14 \\
14\end{array}$ & $\begin{array}{l}8 \\
0.8\end{array}$ & $\begin{array}{l}2 \\
0.2\end{array}$ & $\begin{array}{l}6.3 \times 10^{-8} \\
3.8 \times 10^{-8}\end{array}$ & $\begin{array}{l}2.1 \times 10^{-8} \\
5.6 \times 10^{-9}\end{array}$ \\
\hline
\end{tabular}

\footnotetext{
${ }^{\mathrm{a}}$ For $10^{4}: 1 \gamma$ discrimination efficiency.
}

b Including veto. 
veto efficiency of $50 \%$ can be realistically achieved for internal neutrons. A $\gamma$-ray veto efficiency of $\simeq 70 \%$ has been deemed possible [11]. A relatively modest factor of 2 in WIMP sensitivity can be gained in this way, assuming the veto itself does not contribute to neutron and $\gamma$ backgrounds.

A very worthwhile planned upgrade is the replacement of the phototubes with newly-developed low-radioactivity ones. A 10-fold reduction in background (neutrons and $\gamma$-rays) can be realistically achieved. This would make PMT neutron rates in the target comparable to external neutron backgrounds, pushing the WIMP-nucleon crosssection sensitivity down to $\sim 5 \times 10^{-9} \mathrm{pb}$. With these two upgrades in place ZEPLIN-III would compete favourably with much larger targets and more expensive technologies being considered around the world.

\section{Conclusion}

The ZEPLIN-III performance as a WIMP detector has been assessed using a fully-featured, realistic simulation tool based on GEANT4. The original sensitivity of a few times $10^{-8} \mathrm{pb}$ is confirmed and many aspects of the detector performance have been predicted in anticipation of tests now being carried out in the laboratory.

Some caution must be exercised in interpreting some of these results, notably the discrimination efficiencies and the sensitivity limits derived from them. It is hard to conceive a simulation model which could accurately characterise the tails of the $\gamma$ and recoil distributions to better than 1 part in $10^{4}$. However, most parameters were conservatively chosen, and the real performance could actually surpass that predicted in this work. We shall now discuss some of these uncertainties.

The reference light yield is likely to be higher than $3.4 \mathrm{phe} / \mathrm{keV}$ : early results from our ZEPLIN-II detector (now operating underground) as well as dedicated tests using ZEPLIN-III during its first commissioning run at Imperial College suggest that both the scintillation yield and the photon absorption length in LXe are higher than considered in this simulation, perhaps due to the outstanding purity levels required for charge drift in these large chambers. This will translate directly into an improvement in energy threshold.

New experimental evidence [34] is pointing to larger ionisation yields from nuclear recoils than originally anticipated, more in line with the high-yield scenario considered here. Significantly, it appears that the WIMP sensitivity is remarkably unaffected, at least in this energy range.

A large ionisation Fano factor can make LXe less attractive as a WIMP target, especially if combined with high charge yield. This combination is unlikely, but $F$ is not really known for the electron and nuclear recoil energies of interest.

The new recoil ionisation data also reveal a weak dependence on electric field above $\sim 1 \mathrm{kV} / \mathrm{cm}$ (unlike that indicated in Fig. 4a). Given that charge extraction from electron recoils is still increasing at that field strength, the advantage of high-field operation may prove very significant. Moreover, it is possible that a higher field may decrease the Fano factor and lead to better discrimination, simply because more charge is extracted from the interaction site.

As it stands, ZEPLIN-III should be able to produce a world-beating sensitivity before rival systems and other technologies. With a further upgrade of the phototubes a very significant gain in sensitivity can be achieved, since these dominate both the neutron and $\gamma$-ray backgrounds.

\section{Acknowledgements}

This work has been funded by the UK Particle Physics and Astronomy Research Council (PPARC). Thanks are also due the GEANT4 Collaboration for excellent user support.

\section{References}

[1] T.J. Sumner, New Astron. Rev. 49 (2005) 277-281.

[2] H.M. Araújo, in: Proc. Int. Conf. Dielectric Liquids, Coimbra, Portugal, IEEE Press, New York, 2005, p. 305 (IEEE Cat. 05CH37643)

[3] G.J. Alner et al., New Astron. Rev. 49 (2005) 259-263.

[4] G.J. Alner et al., Nucl. Instrum. Meth. A 555 (2005) 173-183.

[5] B.A. Dolgoshein, V.N. Lebedenko, B.U. Rodionov, JETP Lett. 11 (1970) 513.

[6] T.J. Sumner et al., in: D. Kieda, M. Salamon, B. Dingus, Proc. 26th Int. Cosmic Ray Conf., second ed., 1999, p. 516.

[7] A.S. Howard et al., in: N.J.C. Spooner, V. Kudryavtsev (Eds.), Proc. 3rd Int. Workshop on the Identification of Dark Matter, World Scientific, Singapore, 2001, p. 457.

[8] D.Yu. Akimov et al., in: N.J.C. Spooner, V. Kudryavtsev (Eds.), Proc. 4th Int. Workshop on the Identification of Dark Matter, World Scientific, Singapore, 2003, p. 371.

[9] H. Araújo, ZepIII: A (GEANT4) simulation tool for the ZEPLIN-III detector, ICL Internal Report, 2003

[10] Geant4 Collaboration, Nucl. Instrum. Meth. A 506 (2005) 250.

[11] D. Davidge, Ph.D. Thesis, University of London, 2003.

[12] J. Dawson, Ph.D. Thesis, University of London, 2003.

[13] A. Howard, H. Araújo, 'underground physics' GEANT4 Advanced Example, 2000.

[14] D. Akimov et al., Astroparticle Phys., submitted for publication (ArXiv: astro-ph/0605500).

[15] V.N. Solovov et al., Nucl. Instrum. Meth. A 516 (2004) 462-474.

[16] G.M. Seidel, R.E. Lanou, W. Yao, Nucl. Instrum. Meth. A 489 (2002) 189-194.

[17] D.R. Lide (Ed.), CRC Handbook of Chemistry and Physics, 86th ed., 2005, pp. 12-119.

[18] A. Levin, C. Moisan, TRIUMF Preprint TRI-PP-96-64, GEANT4 User's Guide for Application Developers Section 5.2, 1996. Available from: <http://www.cern.ch/geant4 $>$.

[19] M.E. Moorhead, N.W. Tanner, Nucl. Instrum. Meth. A 378 (1996) $162-170$

[20] H.M. Araújo et al., Nucl. Instrum. Meth. A 521 (2004) 407-415.

[21] ANSYS Inc., www.ansys.com.

[22] T. Doke, K. Masuda, Nucl. Instrum. Meth. A 420 (1999) 62-80.

[23] V. Chepel, M.I. Lopes, V. Solovov, Rad. Phys. Chem. 74 (2005) 160 167.

[24] S. Kubota et al., Phys. Rev. B 20 (8) (1979) 3486-3496.

[25] A. Hitachi et al., Phys. Rev. B 27 (9) (1979) 5279-5285. 
[26] D. Akimov et al., Phys. Lett. B 524 (1979) 245-251.

[27] G.J. Alner et al., Astroparticle Phys. 23 (5) (2005) 444-462.

[28] V. Chepel et al., Astroparticle Phys., in press, doi:10.1016/j.astropartphys.2006.05.001.

[29] E. Aprile et al., Phys. Rev. D 72 (2005) 072006.

[30] J. Dawson et al., Nucl. Instrum. Meth. A 545 (2005) 690-698.

[31] E. Conti et al., Phys. Rev. B 68 (2003) 054201.

[32] T. Takahashi et al., Phys. Rev. A 12 (1975) 1771.

[33] T.Ya. Voronova et al., Sov. Phys. Tech. Phys. 34 (7) (1989) 825-827.

[34] E. Aprile et al., 2006. (ArXiv: astro-ph/0601552).

[35] L.S. Miller et al., Phys. Rev. 166 (3) (1968) 871-878.

[36] T. Doke, Nucl. Instrum. Meth. 196 (1) (1982) 87-96.

[37] E.M. Gushchin et al., Sov. Phys. JETP. 49 (5) (1979) 856-858.

[38] F.P. Santos et al., J. Phys. D: Appl. Phys. 27 (1994) 42-48.

[39] D.Yu. Akimov et al., Phys. At. Nuc. 61 (7) (1998) 1241-1244.

[40] A. Parsons et al., IEEE Trans. Nucl. Sci. 36 (1) (1989) 931-935.

[41] H.N. Ngoc et al., Nucl. Instrum. Meth. A 158 (1979) 128.

[42] A.C. Fonseca et al., Conf. Rec. 2004 IEEE Nucl. Sci. Symp. and Medical Imaging Conf., Rome, Italy, 2004 (IEEE Press, Cat. 04CH37604C, 2005), N16-78.

[43] J. Thomas, D.A. Imel, S. Biller, Phys. Rev. A 38 (1988) 5793.

[44] A.G. Wright, Nucl. Instrum. Meth. A 504 (2003) 245-249.
[45] W.H. Press et al., Numerical Recipes in C: The Art of Scientific Computing, second ed., Cambridge University Press, Cambridge, 1992.

[46] A. Lindote et al., Proc. 7th Int. Conf. Position Sensitive Detectors, 12-16 Sept. 2005, Liverpool, UK, Nucl. Instrum. and Meth. A, to appear.

[47] V.A. Ditlov et al., in: N.J.C. Spooner, V. Kudryavtsev (Eds.), Proc. 4th Int. Workshop on the Identification of Dark Matter, World Scientific, Singapore, 2002, p. 383.

[48] J.W. Marsh et al., Nucl. Instrum. Meth. A 366 (1995) 340-348.

[49] UKDMC public server, Radioactivity data from Nuclear Data Sheets to vol. 64, 1991. Available from: <http://hepwww.rl.ac.uk/ukdmc/ Radioactivity>.

[50] M.J. Carson et al., Nucl. Instrum. Meth. A 548 (2005) 418-426.

[51] P.F. Smith, UKDMC internal note, 2005.

[52] M.J. Carson et al., Astroparticle Phys. 21 (6) (2004) 667-687.

[53] H.M. Araújo, V.A. Kudryavtsev, N.J.C. Spooner, T.J. Sumner, Nucl. Instrum. Meth. A 545 (2005) 398-411.

[54] C. Bungau et al., Astroparticle Phys. 23 (1) (2005) 97-115.

[55] W.B. Wilson et al., 1999, SOURCES-4A Technical Report 13639-LA, Los Alamos.

[56] G.J. Alner et al., Phys. Lett. B 616 (2005) 17-24. 